\section{Following frogs as they flee}

One idea proposed to help species cope with climate change is to ensure that habitat corridors remain available to allow animals to shift their ranges. But a study suggests that some species may still become endangered in a warmer world, even with suitable space to range.

Regan Early at the University of Évora in

Portugal and Dov Sax at Brown University in Providence, Rhode Island, analysed the path that 15 amphibian species in the western United States are likely to have to travel by 2100 to maintain their preferred environmental conditions. The duo found that a key predictor of success was a species' ability to live with unfavourable climate for up to a decade. Furthermore, they say, the variable rate of climate change may mean that some parts of the path exist for too short a time for the amphibians to move through.

The authors conclude that conservationists may need to move certain species, even if habitat corridors do exist. Ecol. Lett. http://dx.doi. org/10.1111/j.14610248.2011.01681.x (2011) BIOMATERIALS

\section{'Braille code' for cell growth}

A material's surface can alter the behaviour of cells sitting on top of it. Researchers looking to make use of this phenomenon have created and screened a wealth of materials with different surface topographies, and identified specific configurations that induce cell growth.

Jan de Boer at the University of Twente in Enschede, the Netherlands, and his colleagues designed chips with 2,176 different topographies. They then grew a type of adult human stem cell on the chips (pictured) and tracked which surface shapes stimulated the cells to multiply or show signs of early bone-cell development.

On the basis of their results, the team developed algorithms that identify which topographic parameters are most likely to trigger certain cell behaviours.

Proc. Natl Acad. Sci. USA http://dx.doi.org/10.1073/ pnas.1109861108(2011)
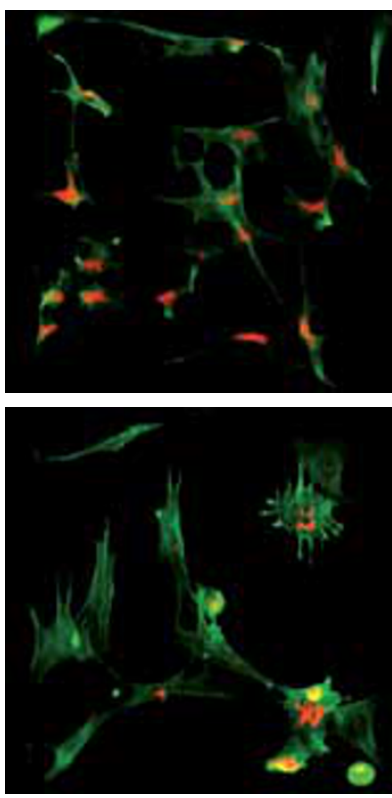

NEUROSCIENCE

\section{Autism in a mouse}

Researchers have created a mouse model of autism by knocking out a gene that is, in humans, associated with a form of the disorder that is often accompanied by epilepsy.

Daniel Geschwind at the University of California, Los Angeles, and his colleagues found that mice lacking the Cntnap2 gene were hyperactive, displayed abnormal social and repetitive behaviours, and had seizures. Certain classes of neurons in the cortex of the animals' brains were in the wrong regions, suggesting abnormal neuronal migration. The brains also had reduced numbers of interneurons cells that provide the major inhibitory signals in the brain.

COMMUNITY CHOICE

The most viewed papers in science

\title{
Why the Atlantic is so hot
}

HIGHLY READ
on www.agu.org
the week of
26 September

The Atlantic Ocean has warmed more than other oceans in recent decades, thanks to the transport of warm waters from the Indian Ocean.

Sang-Ki Lee at the University of Miami in Florida and his colleagues used a global ocean-ice model and historical data to simulate ocean currents, wind patterns and radiative heat fluxes over the course of the late nineteenth and entire twentieth centuries. The models show a sharp increase in heat transport from the Indian Ocean from the 1960s onwards.

The authors say that the strengthening of rotating currents in the Indian and South Atlantic oceans is contributing to this trend. Other research has suggested that the degradation of Antarctic ozone may be contributing indirectly to this oceancurrent strengthening.

Geophys. Res. Lett. http://dx.doi.org/10.1029/2011GL048856 (2011)

The authors suggest that these changes could lead to abnormal neuronal circuits, which could then interfere with neuronal firing and cognitive behaviours.

Treating the mice with the antipsychotic drug risperidone reversed the repetitive behaviours, but had no effect on social behaviour. Cell 147, 235-246 (2011)

\section{ZOOLOG}

\section{How sounds make goldfish skittish}

Startled goldfish dart away from sudden sounds because of sensory organs along the animals' flanks that sense sound vibrations and communicate with a pair of neurons in the brain.

Donald Faber and his colleagues at the Albert Einstein College of Medicine in New York report that nerve cells in the lateral line of goldfish (Carassius auratus; pictured) detect sounds as vibrations.
They then transmit these signals to a single pair of Mauthner cells in the brain in less than a millisecond. Each Mauthner cell triggers muscle contractions along one side of the body, directing the fish away from the sound.

When the researchers disabled the fishes' lateral lines, their escape responses lost all directionality. However, blindfolding fish did not affect their directional behaviour, suggesting that the animals rely mainly on the lateral line to localize the source of sudden sounds. J. Exp. Biol. 214, 3358-3367; 3368-3377 (2011) 